\title{
Targeted Treatments for Cognitive and Neurodevelopmental Disorders in Tuberous Sclerosis Complex
}

\author{
Petrus J. de Vries \\ Neurodevelopmental Service (NDS), Cambridgeshire \& Peterborough NHS Foundation Trust \& Developmental Psychiatry \\ Section, University of Cambridge, Cambridge, CB2 8AH United Kingdom
}

Summary: Until recently, the neuropsychiatric phenotype of tuberous sclerosis complex (TSC) was presumed to be caused by the structural brain abnormalities and/or seizures seen in the disorder. However, advances in the molecular biology of the disorder have shown that TSC is a mammalian target of rapamycin (mTOR) overactivation syndrome, and that direct molecular pathways exist between gene mutation and cognitive/ neurodevelopmental phenotype. Molecularly-targeted treatments using mTOR inhibitors (such as rapamycin) are showing great promise for the physical and neurological phenotype of TSC. Pre-clinical and early-phase clinical studies of the cognitive and neurodevelopmental features of TSC suggest that some of the neuropsychiatric phenotypes might also be reversible, even in adults with the disorder. TSC, fragile $\mathrm{X}$, neurofibromatosis type
1 , and disorders associated with phosphatase and tensin homo (PTEN) mutations, all signal through the mTOR signaling pathway, with the TSC1-TSC2 protein complex as a molecular switchboard at its center. Together, these disorders represent as much as $14 \%$ of autism spectrum disorders (ASD). Therefore, we suggest that this signaling pathway is a key to the underlying pathophysiology of a significant subset of individuals with ASD. The study of molecularly targeted treatments in TSC and related disorders, therefore, may be of scientific and clinical value not only to those with TSC, but to a larger population that may have a neuropsychiatric phenotype attributable to mTOR overactivation or dysregulation. Key Words: TSC, mTOR, rapamycin, neurocognition, memory, autism, autism spectrum disorders.

\section{TUBEROUS SCLEROSIS COMPLEX: THE DISORDER}

\section{Background on tuberous sclerosis complex}

Tuberous sclerosis complex (TSC) is a multisystem genetic disorder caused by a pathogenic mutation in either the TSC1 gene (9q34) or the TSC2 gene (16p13.3). ${ }^{1,2}$ TSC1 and TSC2 lead to essentially identical phenotypic manifestations, although there have been some suggestions that the TSC2 phenotype is typically more severe. ${ }^{3,4}$ The diagnosis is based on a set of clinical criteria ${ }^{5}$ that include so-called "major" and "minor" features. Virtually every organ system in the body can be affected, but major features include facial angiofibromas, cortical tubers, subependymal nodules, cardiac rhabdomyomas, renal angiomyolipomas (AML), and lymphangioleiomyomatosis (LAM) of the lung. ${ }^{1,5}$ There is great variability of expression with some individuals very severely af-

Address correspondence and reprint requests to: Petrus J. de Vries, M.B.Ch.B., M.R.C.Psych., Ph.D., Developmental Psychiatry Section, University of Cambridge, Douglas House, 18b Trumpington Road, Cambridge, CB2 8AH UK. E-mail: pd215@cam.ac.uk. fected by the physical manifestations, whereas others may be relatively mildly affected. ${ }^{1,2}$

The central nervous system manifestations include radiologically detectable structural abnormalities, such as the classic cortical tubers and subependymal nodules, ${ }^{1,2}$ but also include widespread fine-grained white and grey matter abnormalities, ${ }^{6,7}$ which are only detectable through computational morphometric analysis techniques. In 5 to $10 \%$ of subjects, subependymal nodules start to grow and develop into subependymal giant cell astrocytomas (SEGA), typically in the region of the foramen of Monro. ${ }^{1,8,9}$ SEGAs are associated with high rates of morbidity and increased mortality, and typically they require surgical management. ${ }^{1,8,9}$ Seizure disorders are seen in approximately $70 \%$ of individuals with TSC at some stage in their lives. All types of seizures are seen, often in combination; however, in particular, infantile spasms are strongly associated with TSC. ${ }^{1,10}$

\section{The neuropsychiatry of TSC}

The neuropsychiatry of TSC is highly complex and often leads to the most problematic clinical challenges for families. ${ }^{11,12}$ Approximately $30 \%$ of individuals have profound intellectual disability with developmental quotients below 
20. The remaining $70 \%$ fall on a normal distribution of intellectual ability ranging from mild to moderate intellectual disability to superior intelligence. ${ }^{13,14}$ We have recommended referring to these highly distinct groups as the "profound phenotype" and the "normal distribution phenotype" to distinguish clearly between them. ${ }^{14}$ Half of individuals with TSC have normal intelligence (intelligence quotient $>80),{ }^{13}$ but almost none are free from neuropsychiatric problems.

Approximately $25 \%$ of those with TSC meet criteria for autism as defined in International Classification of Diseases-10 or Diagnostic and Statistical Manual of Mental Disorders-IV, ${ }^{15}$ and a total of 40 to $50 \%$ meet criteria for autism spectrum disorder (ASD). ${ }^{15}$ TSC is clearly recognized as one of the medical conditions most highly associated with autism. ${ }^{16}$ Even in those with a normal IQ, autism is approximately 100 times over-represented in comparison with rates in the general population. ${ }^{17}$ No systematic data are available to confirm whether autism in TSC is phenotypically similar to or different from "idiopathic" ASD. However, the clinical impression suggests that the range and features of ASD in TSC are varied, from classic infantile autism to subtle ASD. Attention deficit hyperactivity disorder is seen in approximately $50 \%$ of individuals with TSC, with rates in the range of $30 \%$ in those with a normal IQ, which is approximately 10 times higher than the general population. ${ }^{12,17}$ In adults, the rates of mood and anxiety disorders are highly over-represented with 55 to $59 \%$ meeting criteria for anxiety disorder in systematic studies using research diagnostic criteria. ${ }^{18,19}$

Individuals with TSC are also at high risk of a range of neuropsychological deficits, even those with normal IQs. Deficits (defined as performance below the second percentile of a defined test) are seen in the majority of individuals. This is particularly so for dual task deficits, which are seen in 75 to $90 \%$ of children with normal or near-normal intelligence. ${ }^{20}$ More recently, McCartney et al. ${ }^{21}$ identified evidence of subtle but significant unilateral neglect in normally-intelligent adults with TSC, suggesting deficits also in spatial attention. Memory deficits have been reported in 30 to $40 \%$ of adults with TSC and normal intelligence, ${ }^{6}$ and rates are significantly higher in those with lower intellectual abilities. In a study by Ridler, ${ }^{6}$ recognition memory was comparable to that of matched controls, but verbal and visual recall memory was significantly worse in individuals with TSC in comparison to controls. Some of the most significant deficits were seen on the spatial working memory task (SWM) of the Cambridge Neuropsychological Test Automated Battery (CANTAB), a computer-based neuropsychological tool, ${ }^{22}$ in which individuals with TSC showed very high rates of "between error," but very few "within error" mistakes. ${ }^{6}$ A similar pattern is seen in children with TSC (unpublished data). Executive skills (including planning, cognitive flexibility, and other goal-directed behaviors) are also commonly impaired. In a sequential clinic series, Prather et al. ${ }^{12}$ identified executive deficits in $66 \%$ of those able to participate in direct testing.

Taken together, TSC presents a complex neuropsychiatric profile in the majority of patients. Only a small proportion (approximately 10\%) of individuals do not experience significant impairment in daily life because of these manifestations. No TSC-specific treatments have been identified to date, and no existing neuropsychiatric interventions (pharmacological or nonpharmacological) have been studied in TSC populations. The identification of molecular targets and development of targeted treatments for the cognitive and neurodevelopmental disorders are therefore understandably of high priority for families, clinical professionals, and TSC researchers.

\section{Intracellular signalling in TSC}

TSC1 and TSC2 were identified by positional cloning and have been shown to encode large proteins of 1164 amino acids (TSC1 protein, hamartin) and 1807 amino acids (TSC2 protein, tuberin), respectively. ${ }^{1,2,23}$ TSC 1 and TSC2 form a heterodimeric complex that acts as a central player to integrate intracellular and extracellular signals across a number of conserved cell signaling pathways $^{1,2,23}$ (FIG. 1). The TSC1-TSC2 complex has multiple phosphorylation sites and receives direct "upstream" signals from protein kinase B (AKT), glycogen synthase kinase $3 \beta$ (GSK3 $\beta$ ), p38MAPK-activated protein kinase (MK2), extracellular signal-regulated kinase 1 and 2 (ERK1/2), and adenosine monophosphate-activated protein kinase (AMPK). ${ }^{1,2,23}$

The best understood downstream (output) pathway is via the GTPase activating protein (GAP) domain of TSC2. Phosphorylation at several TSC1-TSC2 sites activates the GAP function of TSC2, whereas phosphorylation at other sites inhibits it. ${ }^{1,2,23,24}$ Inhibition of the GAP function shifts the balance of Ras homologue enriched in brain (Rheb) from its Rheb-GDP to Rheb-GTP form, regulating activation of the mTOR complex 1 proteins. mTOR activation leads to phosphorylation of downstream targets including p70S6kinase 1 (S6K1) and eukaryotic initiation factor $4 \mathrm{E}$ binding protein 1 (4E-BP1) that drive protein synthesis and cell proliferation. ${ }^{1,2,16,23,24}$ In TSC, loss of either TSC1 or TSC2 through spontaneous mutation (70\% of cases) or inherited mutation (30\% of cases), and followed by a "second hit" of the remaining functional allele, which therefore prevents formation of the heterodimeric complex, causing loss of regulatory control over mTOR, thus leading to overactive cell growth and proliferation. ${ }^{1,2,23,24}$ Therefore, TSC is an mTOR overactivation syndrome.

The TSC1-TSC2-mTOR signalling pathway as shown in FIG. 1 was generally believed to be conserved throughout all eukaryotic groups. ${ }^{25,26}$ How- 


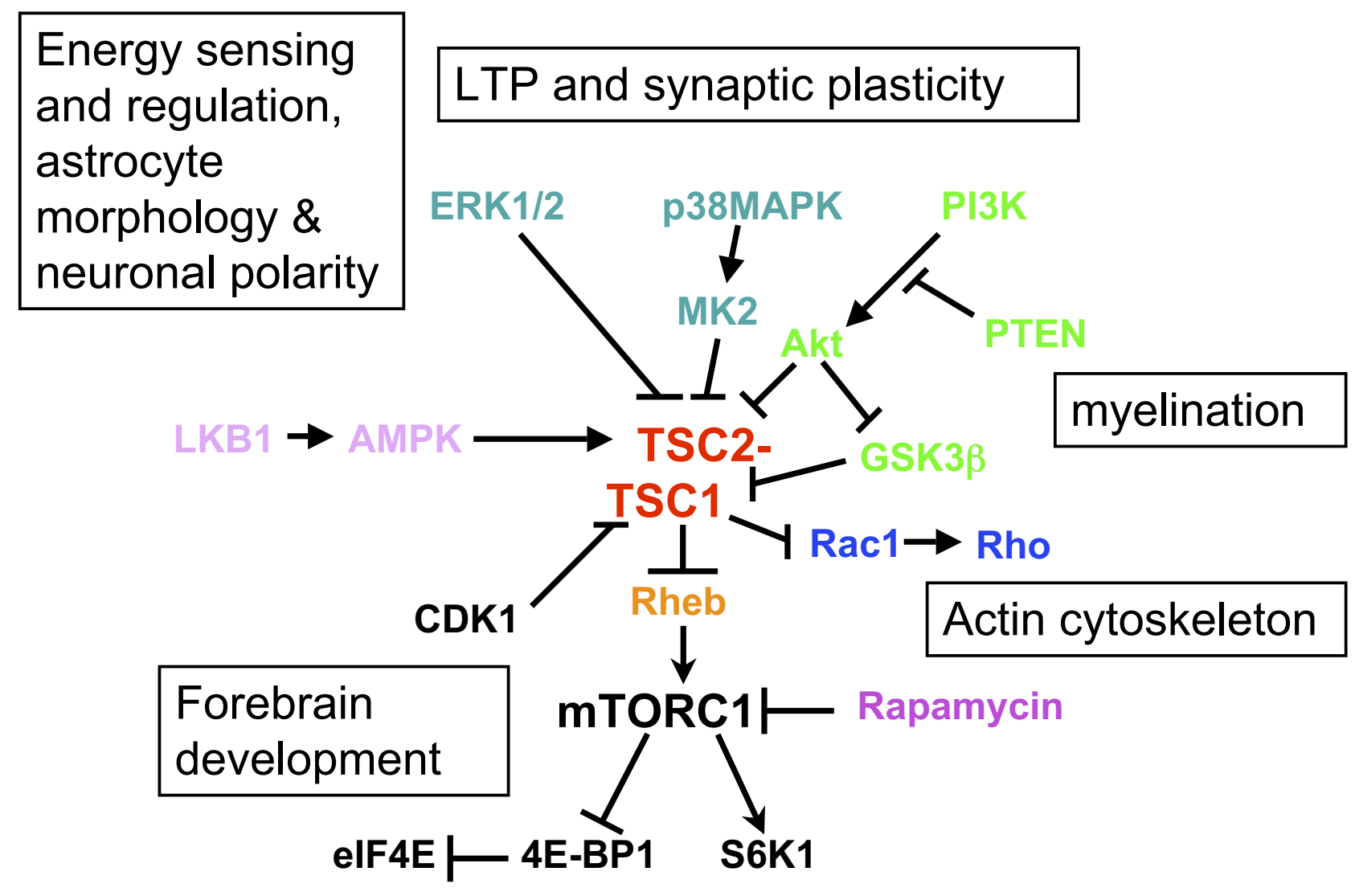

\section{Cell Growth \& Proliferation}

FIG. 1. Intracellular signaling and the neurobiology of tuberous sclerosis complex (TSC). The TSC1-TSC2 complex acts as a heterodimer at the crossroads of a number of important signaling pathways. These include the insulin-signaling PI3K-AKT pathway, the mitogen-activated p38MAPK and ERK1/2 pathways, and the energy-sensing AMPK pathway. Proteins in these pathways have fundamental neurobiological roles as represented schematically in the figure. One of the crucial downstream targets regulated through the TSC1-TSC2 complex is mTORC1, a key regulator of protein synthesis and cell growth. Given that TSC is an mTOR overactivation syndrome, rapamycin and other mTOR inhibitors are the principal current therapeutic tools targeted at reversing/improving physical and neuropsychiatric phenotypes in TSC and related disorders. (For further details, please see the text of the article.) $4 \mathrm{E}-\mathrm{BP} 1=$ eukaryotic initiation factor $4 \mathrm{E}$ binding protein 1 ; AKT = protein kinase B; AMPK = adenosine monophosphate activated protein kinase; CDK1 = cyclin-dependent kinase 1; EIF4E = eukaryotic translation initiation factor 4E; ERK1/2 = extracellular signal regulated kinase 1 and 2; GSK3beta = glycogen synthase kinase 3 beta; LKB1 = serine/threonine kinase 11; LTP = long-term potentiation; MK2 = MAPK-activated protein kinase 2; $\mathrm{mTORC1}=$ mammalian target of rapamycin complex 1; p38MAPK = p38 mitogen-activated protein kinase; PI3K = phosphoinositide 3 kinase; PTEN = phosphatase and tensin homolog; Rac1 = Ras-related C3 botulinum toxin substrate 1; Rheb = Ras homologue enriched in brain; Rho = Ras homologue gene family, member A; S6K1 = ribosomal p70 S6 kinase 1; TSC1 = tuberous sclerosis complex 1 protein (hamartin); TSC2 $=$ tuberous sclerosis complex 2 protein (tuberin).

ever, recent bio-informatic approaches have shown that the pathway was built up from a simpler one, present in the ancestral eukaryote, coupling cell growth and energy levels. Additional elements such as TSC1 and TSC2 were "bolted on" only in particular eukaryotic lineages. ${ }^{27}$ Comparison of sequences across various organisms suggests that not all functional domains seen in humans are conserved in nonhuman homologues, thus raising the need to consider how well the signaling pathway in humans can be modelled using proteins from other organisms. ${ }^{27}$

\section{The neurobiology of TSC}

Until recently, it was believed that the neuropsychiatric manifestations of TSC (notably intellectual disability and autism) were caused by the structural abnormalities (tubers and nodules) and/or by the seizure disorders seen in TSC. ${ }^{28,29}$ The therapeutic implications of these assumptions were that structural abnormalities are unchangeable; therefore, the best treatment strategy would lie in the aggressive treatment of epilepsy or in the prophylactic treatment for seizures, even pre-natally. ${ }^{10,30} \mathrm{Ad}-$ vances in the molecular biology of TSC have fortunately 
identified that the TSC-mTOR signaling pathways are involved in a number of key neurobiological processes, including long-term potentiation (LTP) and synaptic plasticity, myelination, actin cytoskeletal formation, forebrain development, energy sensing, astrocyte morphology, and neuronal polarity, all of which have been clearly shown to contribute to various neuropsychiatric phenotypes. $^{2,11,16,31}$ This realization led de Vries and Howe $^{2}$ to posit a molecular hypothesis for the neuropsychiatric manifestations of TSC, referred to as the Global Regulator and Integrator of Physiological Processes (or GRIPP) hypothesis. Given the range of fundamental neurobiological processes in the TSC-mTOR signaling pathway, de Vries and $\mathrm{Howe}^{2}$ proposed that structural and seizure factors were neither necessary nor sufficient to explain the neuropsychiatric phenotypes observed in TSC, but that a direct molecular route would be sufficient (referred to as GRIPP I, the first part of the hypothesis). On publication of the hypothesis, no TSC animal behavior studies had yet been performed, but a prediction was that TSC animal models might present with neurocognitive deficits, yet be free from structural brain abnormalities and seizures. ${ }^{2}$ This was subsequently confirmed in $\mathrm{Tscl}^{+/-}$and $\mathrm{Tsc}^{+/-}$mouse models, both free from neuropathology and seizures. ${ }^{32,33}$ The $T s c 1^{+/-}$mice showed context discrimination, spatial working memory and socialization deficits. ${ }^{32}$ The $T s c 2^{+/-}$mice showed similar context discrimination and spatial working memory deficits, but no socialization deficits. ${ }^{33}$ Interestingly, on the radial maze task, the animal equivalent of the CANTAB spatial working memory test (see previously), the $T s c 2^{+/-}$mice showed significant "across errors" (equivalent to "between errors" in humans), but no "within error" mistakes ${ }^{33}$ in a pattern highly reminiscent of the human findings. ${ }^{6}$ Therefore, evidence was clearly emerging of a direct molecular route from TSC mutation to neuropsychiatric phenotypes.

The second part of the GRIPP molecular hypothesis stated that the vast range of TSC1 and TSC2 mutations seen in humans with TSC will have a range of consequences at the biochemical and signaling level. ${ }^{2}$ Therefore, GRIPP II predicted that the variability of neurocognitive manifestations observed in TSC would be dependent on the nature of the structural and functional impairment of protein and phosphorylation sites caused by individual mutations. ${ }^{2}$ This might depend on various factors such as stability and activity of truncated or full-length mutant protein, how nonsense-mediated decay might influence the production of truncated proteins in the brain and differential expression of proteins in different brain regions, tissue, or cell types. $^{2}$ To date there is no strong evidence to support GRIPP II, and the current view would be that variability of behavioral phenotypic expression is caused by geneenvironment interactions, modifier genes, and so forth.
GRIPP II does not deny that these may be additional factors, only that they may not be necessary to explain the phenotypic variability observed. There are some emerging biochemical studies that show differential functional effects of different TSC1 and TSC2 mutations. In a study of TSCl missense mutations, Mozzaffari et $\mathrm{al}^{34}$ identified specific substitutions between amino acids 50-90 that resulted in reduced steady state levels of tuberin, leading to increased mTOR signaling. Similarly, a study of different TSC2 mutations have shown distinct effects from one another on tuberin and mTOR function. ${ }^{35}$

\section{HOW IS TSC RELEVANT TO AUTISM?}

Even though the etiology of autism spectrum disorders (ASD) is typically attributed to an oligogenic basis (reviewed by Persico and Bourgeron ${ }^{36}$ ), an increasing number of single gene disorders with very high prevalence of autism are being recognized, representing at least $15 \%$, if not more, of the total rates of ASD. ${ }^{16}$ In a translational medicine commentary, de Vries ${ }^{37}$ recently suggested that the current oligogenic model of autism could be conceptualized as a map of the London underground, where a single-gene mutation is not sufficient to disrupt the neurodevelopmental trajectory and lead to ASD. Therefore, multiple genes (each making a small contribution) are required to be aberrant before ASD would manifest. An oligogenic model does not present any immediate targets for molecular treatment strategies.

The increasing number of single gene disorders associated with ASD, de Vries ${ }^{37}$ proposed, may point to an alternative etiological model of autism, which is more similar to a map of the "Boston T" public transport system where disruption in only one spot (or single gene) along one of a number of distinct tracks might be sufficient to disrupt the neurodevelopmental journey and lead to ASD (FIG. $1^{37}$ ). If this conceptualization were to be correct, then "repair" of that one spot (or biological consequence of a single gene mutation) might be sufficient to improve the neurodevelopmental journey and improve the manifestations of ASD. Therefore, identifying the specific underlying pathophysiological mechanism in a single gene disorder, such as TSC, may point to a group of underlying etiologies (shared lines), which in turn may highlight potential molecular intervention targets not only for the single gene disorder, but also for others in the shared etiological group.

TSC and fragile $X$ are now recognized to have the highest association with autism and ASD. ${ }^{16,38}$ Two other single-gene disorders with high rates of autism include neurofibromatosis type 1 (NF1), in which the rates of autism range between 0 and $4 \%$, and disorders associated with PTEN mutations, representing as much as $1 \%$ of all cases of ASD. ${ }^{16}$ Taken together, these four disorders 
may represent up to $14 \%$ of all cases of ASD. ${ }^{16}$ Therefore, it is highly significant that all four of these disorders signal through the PI3K-TSC1/2-mTOR signaling pathway, with the TSC1-TSC2 complex as a "molecular switchboard" at its center (FIG. 1). PTEN inhibits PI3Kdependent signaling, and loss of PTEN function in neurons leads to overactivation of mTOR complex $1 .{ }^{39}$ The fragile $\mathrm{X}$ mental retardation protein (FMRP) binds to specific mRNAs, repressing their translation. ${ }^{16}$ There is now evidence that lack of FMRP (as seen in fragile X) leads to mTOR overactivation. ${ }^{38}$ The NF1 protein, neurofibromin, functions as a Ras-GTPase activating protein via PI3K-AKT-TSC2 and regulates mTOR. In NF1 deficient cells mTOR is constitutively activated. ${ }^{40}$ Therefore, we suggest that the signaling pathway (as shown in FIG. 1) is an important key to the underlying pathophysiology of a significant subset of individuals with ASD. The study of molecularly-targeted treatments in TSC may therefore be scientifically and clinically valuable not only to those with TSC but to a large population that may have a neuropsychiatric phenotype attributable to mTOR overactivation or dysregulation.

\section{TARGETED TREATMENTS IN TSC}

\section{Treatment of the physical features of TSC}

The molecular understanding of the pathophysiology of peripheral features in TSC has led to targeted interventions using rapamycin to inhibit mTOR (FIG. 1). Rapamycin (or Sirolimus) is a macrolide isolated from a strain of Streptomyces hygroscopicus, which is indigenous to the Easter Islands (also known as "Rapa Nui"). ${ }^{1}$ Rapamycin binds FK-binding protein 12 (FKBP12), and as a complex, rapamycin-FKBP12 directly binds mTOR complex 1, thus reducing phosphorylation of downstream mTOR targets. Rapamycin is well known for its use as an immunosuppressant to prevent organ rejection, particularly after renal transplant, and it has been in clinical use for many years. ${ }^{24} \mathrm{~A}$ number of other mTOR inhibitors are available or under development, including temsirolimus (CCI-779) and everolimus (RAD001).

A couple of pre-clinical trials have shown that rapamycin, temsirolimus, or everolimus reduced renal cyst and tumor development and reduced the volume of existing renal tumors in animal models. ${ }^{41,42}$ Given the considerable experience with rapamycin in human populations, translation of the pre-clinical findings to human clinical trials was rapid. Bissler et $\mathrm{al}^{43}$ reported a singlesite, phase I/II open-label study of rapamycin for the treatment of renal AML in 25 patients with TSC or sporadic LAM. Mean AML volumes were reduced by $53 \%( \pm 26.6 \%)$ from baseline after 12 months of treatment. Interestingly, regrowth to $85 \%( \pm 28.5 \%)$ of baseline volume occurred within the subsequent 12 months after discontinuation of therapy. ${ }^{43}$ The United Kingdom- based multicenter Trial of Efficacy and Safety of Sirolimus in Tuberous Sclerosis and LAM (TESSTAL) trial ${ }^{44}$ reported very similar findings, with an equivalent reduction in AML volume in excess of 50\%. Side effects were seen in both studies, but were similar to those seen in non-TSC post-transplant patients, and the side effects were typically low grade and self-limiting. ${ }^{24,43,44}$ Both studies also examined the efficacy of rapamycin on the lung manifestation, LAM, seen in association with TSC, or in sporadic form. Bissler et al's ${ }^{43}$ study reported some improvement in lung function parameters. However, the TESSTAL trial ${ }^{44}$ did not observe any significant improvements. A number of larger scale trials are currently underway for various aspects of the physical phenotype of TSC, including a phase III trial for renal AML (Examining everolimus in a study of TSC [EXIST]-2 trial; ClinicalTrials.gov; NCT00790400).

A single case study ${ }^{45}$ reported improvement of the characteristic skin lesions (i.e., facial angiofibromas) in a patient with TSC who was taking oral rapamycin. Therefore, preclinical and clinical studies are monitoring skin manifestations in trials of systemic mTOR inhibitors, and are examining topical application of rapamycin in preclinical ${ }^{46}$ and clinical trials (ClinicalTrials.gov; NCT01031901).

\section{Treatment of CNS tumors and seizures in TSC}

Given the high rates of CNS lesions in TSC, Franz et $\mathrm{al}^{9}$ examined the efficacy of oral rapamycin in SEGAs in a case series of four patients with TSC and SEGA. Prior to treatment, all lesions showed growth on serial neuroimaging. After treatment with rapamycin, all lesions showed regression. Interruption of treatment resulted in regrowth of the SEGA in one patient. The treatment was reported as well-tolerated by patients, and suggested that oral mTOR inhibitors may offer an alternative to surgical removal of such lesions. ${ }^{9}$ This case series was followed by a larger open-label phase II study (ClinicalTrials.gov; NCT00411619) with positive interim results. ${ }^{47}$ An international phase III trial is currently underway (EXIST-1; ClinicalTrials.gov; NCT00789828).

As discussed earlier, epilepsy is a common feature in TSC, which can be refractory to available pharmacological treatment options. ${ }^{1,10}$ Seizures are assumed to originate in the peri-tuber regions in the cerebral cortex, but this assumption is not universally accepted..$^{10}$ In particular, examination of the role of mTOR signaling has revealed important mechanistic links to epileptogenesis. ${ }^{10}$ For instance, abnormal cell growth and proliferation due to mTOR overactivation could affect excitability of neuronal circuits to promote seizures; mTOR aberration could lead to abnormal neuronal morphology, synaptic plasticity or affect neurotransmitter, and ion 
channel expression. At least two TSC mouse models ${ }^{48,49}$ have shown abnormal neurological phenotypes, including megalencephaly, neuronal hypertrophy, astrocytosis, and reduced myelination. Importantly, all these abnormalities were reversed or improved by mTOR inhibitors. $^{48,49}$ In a study of a knock-out mouse involving inactivation of the TSCl gene in glial fibrillary acidic protein (GFAP)-positive cells ( $T s c l^{\mathrm{GFAP}} \mathrm{CKO}$ mice), ${ }^{49}$ rapamycin treatment prior to the onset of seizures prevented the subsequent development of epilepsy, whereas treatment after the onset of epilepsy reduced seizure frequency. Importantly, the neurological phenotype emerged or re-emerged after the cessation of rapamycin.

A phase I/II open-label study by Krueger et $\mathrm{al}^{50}$ reported reduction in seizure frequency in a group of young children who were treatment-resistant to conventional anti-epilepsy drugs. Larger scale, placebo-controlled trials are awaited.

\section{Targeted treatment of the cognitive and neurodevelopmental disorders in TSC}

Advances in the molecular neurobiology of TSC have opened up exciting possibilities for moleculartargeted treatments of the neuropsychiatric phenotype in TSC. The main treatment implication of the molecular "GRIPP" hypothesis ${ }^{2}$ was this: if molecular aberrations could directly lead to neurocognitive manifestations, appropriate molecular targeting of those deficits could reverse or improve the neurocognitive deficit. Therefore, if heterozygous models of TSC1 and TSC2, free from structural and electrophysiological abnormalities, showed neurocognitive deficits, de Vries and Howe ${ }^{2}$ predicted that rapamycin would act as a cognitive enhancer by reducing the effects of mTOR overactivation.

Using a $T s c 2^{+/-}$mouse model, Ehninger et $\mathrm{al}^{33}$ showed deficits in learning and memory in adult mice, in the absence of neuropathology or seizures. They showed that overeractive mTOR signaling in the hippocampus led to abnormal long-term potentiation in the CA1 region of the hippocampus and consequently to deficits on two spatial learning and one context discrimination task. After intraperitoneal administration of rapamycin for up to 5 days, synaptic plasticity abnormalities, as well as the learning/memory deficits (i.e., water maze and context discrimination), were reversed to be indistinguishable from wild-type mice. ${ }^{33}$ The group unfortunately were not able to examine the effect of rapamycin on the radial maze task, which showed interesting similarity to human cognitive profiles. No rapamycin rescue studies examining behavioral phenotypes have been performed on the $\mathrm{TsCl}^{+/-}$mice or any other TSC animal models to date. In particular, no study has examined the effect of mTOR inhibition on socialization behaviors that is presumed to be of particular significance to ASD phenotypes in humans.
Given the theoretical rationale for potential cognitive enhancement, and given findings in the literature suggesting impairment of memory recall in wild-type animals after administration of rapamycin, ${ }^{51,52}$ we added measures of learning and memory as secondary outcomes to the multicenter TESSTAL trial (ClinicalTrials. gov; NCT004907789). Measures included a list learning task, a story recall task, and a figure recall task. Assessments were performed at baseline, and at 4 months and 12 months after the initiation of rapamycin. After 4 months, measures of immediate and delayed recall improved by $20 \%$ or more from baseline in five of the eight subjects with TSC in the trial. ${ }^{53}$ One subject did not show any clinical response. Interestingly, two subjects showed deterioration on some (but not all) of the measures. The 12-month data were not yet available at the time of this publication. It will be of great interest to see if positive changes were sustained between 4 and 12 months, and whether additional, later changes may have been observed.

Although these findings were clearly preliminary, results raised some interesting and important issues for consideration in future trials. The positive changes observed in the human trial matched the findings from the $T s c 2^{+/-}$mouse study. ${ }^{33}$ However, Ehninger et $\mathrm{al}^{33}$ did not observe any negative change. Therefore, a priority of translational neuroscience research in TSC should be to identify predictors of likely positive or negative changes. A number of possible contributors may exist: (1) side effects may have influenced compliance of subjects resulting in different durations or doses of rapamycin, (2) trough levels of rapamycin may have been different between subjects and may contribute to the neurocognitive efficacy of mTOR inhibitors, (3) different genotypes may have differential patterns of mTOR overactivation and may have responded differently to rapamycin as a consequence, and (4) subjects in the trial differed in neurocognitive performance at baseline, and it is not unreasonable to predict that ability at baseline might influence the likely direction of change in response to an mTOR inhibitor.

In their review of single-gene contributors to autism, Kelleher and Bear ${ }^{16}$ suggested that bidirectional alterations in synaptic protein synthesis might be seen in autism (see Figure 2 in Kelleher and Bear ${ }^{16}$ ). Disorders such as fragile $\mathrm{X}$ and TSC were proposed to lead to overactive synaptic protein synthesis and to "hyperconnectivity" in neuronal networks. In contrast, Rett's syndrome was proposed to lead to reduced synaptic protein synthesis, thus leading to neuronal "hypoconnectivity." We suggest that the association between mTOR and neurocognitive performance may also not be linear, even within a single syndrome such as TSC.

The $T s c 2^{+/-}$mouse article ${ }^{33}$ led to enormous interest in the scientific and lay community alike. It is important 
to be mindful that no studies to date have directly examined the relationship between an autism phenotype and rapamycin. Similarly, no studies to date have shown (outside the TSC/fragile X/NF1/PTEN cluster of disorders) whether any other autism phenotypes are associated with mTOR dysregulation. This would be an essential first step prior to considering trials of mTOR inhibitors in ASD in the absence of any of the previously mentioned single-gene disorders.

Many unanswered questions remain: What would happen after discontinuation of mTOR inhibitors? What will the clinical impact be to those who are most impaired? Which groups of individuals are most likely to benefit? Can we identify biomarkers of likely response? In spite of the many outstanding questions and the limited animal and human data, there remains significant enthusiasm for the potential neuroscientific application of mTOR inhibitors. Large-scale phase I/II clinical trials are currently being set up in the United Kingdom and the United States to examine the impact of mTOR inhibition on the cognitive and neurodevelopmental manifestations, including autism, in TSC.

\section{CONCLUSION}

Less than a decade ago, single-gene disorders, such as TSC, were considered scientific curiosities, with relevance only to themselves. However, advances in the molecular biology of these disorders have opened up an exciting scientific landscape to explore molecular-targeted treatments and to translate them into human settings with implications far beyond the boundaries of individual disorders. Many challenges remain for the translational neuroscience of genetic disorders, but the paradigm shift that neuropsychiatric phenotypes may be reversible (even in adults) has occurred.

\section{Disclosure}

This article was supported in part by funding from the Cambridgeshire \& Peterborough NHS Foundation Trust, the Tuberous Sclerosis Association (UK) and the TSAlliance (US). Dr. de Vries is on the international steering committee of EXIST-1 and EXIST-2 trials, funded by Novartis, but he does not receive any fees from Novartis.

\section{REFERENCES}

1. Crino PB, Nathanson KL, Henske EP. The tuberous sclerosis complex. N Engl J Med 2006;355:1345-1356.

2. de Vries PJ, Howe CJ. The tuberous sclerosis complex proteins-a GRIPP on cognition and neurodevelopment. Trends Mol Med 2007;13:319-326.

3. Jones AC. Molecular genetic and phenotypic analysis reveals differences between TSC 1 and TSC2 associated familial and sporadic tuberous sclerosis. Hum Mol Genet 1997;6:2155-2161.

4. Dabora SL. Mutational analysis in a cohort of 224 tuberous sclerosis patients indicates increased severity of TSC2, compared with TSC1, disease in multiple organs. Am J Hum Genet 2001;68:64-80.
5. Roach ES, Gomez MR, Northrup H. Tuberous sclerosis consensus conference: revised diagnostic criteria. J Child Neurol 1998;13: $624-628$

6. Ridler K. Neuroanatomical correlates of memory deficits in tuberous sclerosis complex. Cereb Cortex 2007;17:261-271.

7. Ridler K. Widespread anatomical abnormalities of grey and white matter structure in tuberous sclerosis. Psychol Med 2001;31:14371446.

8. Franz DN, de Vries PJ, Crino PB. Giant cell astrocytomas in tuberous sclerosis complex. Arch Dis Child 2009;94:75-76.

9. Franz DN, Leonard J, Tudor C, et al. Rapamycin causes regression of astrocytomas in tuberous sclerosis complex. Ann Neurol 2006; 59:490-498.

10. Wong M. Mammalian target of rapamycin (mTOR) inhibition as a potential antiepileptogenic therapy: from tuberous sclerosis to common acquired epilepsies. Epilepsia 2010;51:27-36.

11. Ehninger D, de Vries PJ, Silva AJ. From mTOR to cognition: molecular and cellular mechanisms of cognitive impairment in tuberous sclerosis. J Intellect Disabil Res 2009;53:838-851.

12. Prather P, de Vries PJ. Behavioral and cognitive aspects of tuberous sclerosis complex. J Child Neurol 2004;19:666-674.

13. Joinson C. Learning disability and epilepsy in an epidemiological sample of individuals with tuberous sclerosis complex. Psychol Med 2003;33:335-344.

14. de Vries PJ, Prather P. The tuberous sclerosis complex. N Engl J Med 2007;356:92.

15. Bolton PF, Park RJ, Higgins NP, Griffiths PD, Pickles A. Neuroepileptic determinants of autism spectrum disorders in tuberous sclerosis complex. Brain 2002;125:1247-1255.

16. Kelleher RJ, Bear MF. The autistic neuron: troubled translation? Cell 2008;135:401-406.

17. de Vries PJ, Hunt A, Bolton PF. The psychopathologies of children and adolescents with tuberous sclerosis complex (TSC): a postal survey of UK families. Eur Child Adolesc Psychiatry 2006;16:16-24.

18. Lewis JC, Thomas HV, Murphy KC, Sampson JR. Genotype and psychological phenotype in tuberous sclerosis. J Med Genet 2004; 41:203-207.

19. Smalley SL, Burger F, Smith M. Phenotypic variation of tuberous sclerosis in a single extended kindred. J Med Genet 1994;31:761765

20. de Vries PJ, Gardiner J, Bolton PF. Neuropsychological attention deficits in tuberous sclerosis complex (TSC). Am J Med Genet Part A 2009;149A:387-395.

21. McCartney DL, Bullmore ET, Suckling J, et al. Lateralised spatial attentional bias and white matter tract connectivity in tuberous sclerosis complex (TSC). J Intell Disabil Res 2009;53:836.

22. Sahakian BJ, Owen AM. Computerized assessment in neuropsychiatry using CANTAB: discussion paper. J Royal Soc Med 1992; 85:399-402.

23. Kwiatkowski DJ, Manning BD. Tuberous sclerosis: a GAP at the crossroads of multiple signalling pathways. Hum Mol Genet 2005; 14:R251-R258.

24. Sampson JR. Therapeutic targeting of mTOR in tuberous sclerosis. Biochem Soc Trans 2009;37:259-264.

25. Jacinto ER, Loewith A, Schmidt S, et al. Mammalian TOR complex 2 controls the actin cytoskeleton and is rapamycin insensitive. Nature Cell Biol 2004;6:1122-1128.

26. Gwinn DM, Shackelford DB, Egan DF, et al. AMPK phosphorylation of raptor mediates a metabolic checkpoint. Mol Cell 2008; 30:214-226.

27. Serfontein J, Nisbet RER, Howe CJ, de Vries PJ. Evolution of the TSC1/TSC2-TOR signalling pathway. Sci Signal 2010;3:ra49.

28. O'Callaghan FJK, Harris T, Joinson C, et al. The relation of infantile spasms, tubers, and intelligence in tuberous sclerosis complex. Arch Dis Child 2004;89:530-533.

29. Raznahan A, Higgins NP, Griffiths PD, Humphrey A, Yates JRW, Bolton PF. Biological markers of intellectual disability in tuberous sclerosis. Psychol Med 2007;37:1293-1304.

30. Jozwiak S, Domanska-Pakiela D, Kotulska K, et al. New indications for antiepileptic drugs application in infants with tuberous sclerosis complex - prevention of epilepsy. Pharmacol Reports 2009;61:580-581. 
31. Costa-Mattioli M, Sossin WS, Klann E, Sonenberg N. Translational control of long-lasting synaptic plasticity and memory. Neuron 2009;61:10-26.

32. Goorden SMI, van Woerden GM, van der Weerd L, Cheadle JP, Elgersma Y. Cognitive deficits in $T s c 1^{+1-}$ mice in the absence of cerebral lesions and seizures. Ann Neurol 2007;62:648-655.

33. Ehninger D, Han S, Shilyansky C, et al. Reversal of learning deficits in a $T s c 2^{+/-}$mouse model of tuberous sclerosis. Nat Med 2008; 14:843-848.

34. Mozzaffari M, Hoogeveen-Westerveld M, Kwiatkowski D, et al. Identification of a region required for TSC 1 stability by functional analysis of TSC1 missense mutations found in individuals with tuberous sclerosis complex. BMC Med Genet 2009;10:88.

35. Nellist M, Sancak O, Goedbloed MA, et al. Distinct effects of single amino-acid changes to tuberin on the function of the tuberinhamartin complex. Eur J Hum Genet 2005;13:59-68.

36. Persico AM, Bourgeron T. Searching for ways out of the autism maze: genetic, epigenetic and environmental clues. Trends Neurosci 2006;29:349-358.

37. de Vries PJ. Genetics and neuropsychiatric disorders: genomewide, yet narrow. Nat Med 2009;15:850-851.

38. Wang LW, Berry-Kravis E, Hagerman RJ. Fragile X: leading the way for targeted treatments in autism. Neurotherapeutics 2010;7: 264-274.

39. Kwon CH, Luikart BW, Powell CM, et al. PTEN regulates neuronal arborisation and social interaction in mice. Neuron 2006;50: $377-388$.

40. Johannessen CM, Reczek EE, James MF, Brems H, Legius E, Cichowski K. The NF1 tumor suppressor critically regulates TSC2 and mTOR. Proc Natl Acad Sci USA 2005;102:85738578.

41. Kenerson H, Dundon TA, Yeung RS. Effects of rapamycin in the Eker rat model of tuberous sclerosis complex. Pediatr Res 2005; 57:67-75.

42. Lee L, Sudentas P, Donohue B, et al. Efficacy of a rapamycin analog (CCI-779) and IFN- $\gamma$ in tuberous sclerosis mouse models. Genes Chromosomes Cancer 2005;42:213-227.

43. Bissler JJ, McCormack FX, Young LR, et al. Sirolimus for angiomyolipoma in tuberous sclerosis complex or lymphangioleiomyomatosis. N Engl J Med 2008;358:140-151.
44. Davies DM, Johnson SR, Tattersfield AE, et al. Sirolimus therapy in tuberous sclerosis or sporadic lymphangioleiomyomatosis. N Engl J Med 2008;358:200-203.

45. Hofbauer GFL, Marcollo-Pini A, Corsenca A, et al. The mTOR inhibitor rapamycin significantly improves facial angiofibroma lesions in a patient with tuberous sclerosis. B J Dermatol 2008;159: 473-475.

46. Rauktys A, Lee N, Lee L, Dabora SL. Topical rapamycin inhibits tuberous sclerosis tumor growth in a nude mouse model. BMC Dermatol 2008;8:1.

47. Krueger DA, Care MM, Agricola K, et al. Safety and efficacy of RAD001 (everolimus) for the treatment of subependymal giant cell astrocytoma in pediatric and adult patients with TSC. Presented at the TSC International Research Conference, University of Sussex; September 11-13, 2008; Brighton, UK.

48. Zeng L-H, Xu L, Gutmann DH, Wong M. Rapamycin prevents epilepsy in a mouse model of tuberous sclerosis complex. Ann Neurol 2008;63:444-453.

49. Meikle L, Pillizzi K, Egnor A, et al. Response of a neuronal model of tuberous sclerosis to mammalian target of rapamycin (mTOR) inhibitors: effects on mTORC1 and Akt signalling lead to improved survival and function. J Neurosci 2008;28:54225432.

50. Krueger DA, Holland-Bouley K, Byers AW, et al. Effect of RAD001 (everolimus) on epilepsy and neurocognition in patients with TSC. Presented at the TSC International Research Conference, University of Sussex; September 11-13, 2008; Brighton, UK.

51. Chen X, Garelick MG, Wang HB, Athos J, Storm DR. PI3 kinase signalling is required for retrieval and extinction of contextual memory. Nat Neurosci 2005;8:925-931.

52. Dash PK, Orsi SA, Moore AN. Spatial memory formation and memory-enhancing effect of glucose involves activation of the tuberous sclerosis complex-mammalian target of rapamycin pathway. J Neurosci 2006;26:8048-8056.

53. de Vries PJ. The Neuroscience of TSC - are we at risk of getting lost between the bench and the bedside? Presented at the International TSC Research Conference: From DNA to Human Therapies; September 23-26, 2009; Chicago. 